\title{
Impact of Free Maternity Services on Outcomes related to Hypertensive Disorders of Pregnancy at Moi Teaching and Referral Hospital in Kenya: a retrospective analysis
}

Marie Buitendyk ( $\nabla$ marie.buitendyk@medportal.ca )

University of Toronto

Wycliffe Kosgei

Moi University

Julie Thorne

University of Toronto

Heather Millar

University of Toronto

Joy Marsha

Moi University

Vincent Kibet

Moi University

Christian Ochieng Bernard

Moi University

\section{Beth Payne}

University of British Columbia

Caitlin Bernard

Indiana University

\section{Astrid Christoffersen-Deb}

University of British Columbia

\section{Research Article}

Keywords: hypertensive disorders of pregnancy, preeclampsia, eclampsia, global health, maternal mortality, neonatal mortality

Posted Date: January 5th, 2021

DOI: https://doi.org/10.21203/rs.3.rs-135065/v1 
License: (c) (i) This work is licensed under a Creative Commons Attribution 4.0 International License. Read Full License 


\section{Abstract \\ Background}

Preeclampsia is a major contributor to maternal and neonatal mortality worldwide. Ninety-nine percent of these deaths occur in resource limited settings. One of the greatest barriers to women seeking medical attention remains the cost of care. Kenya implemented a nation-wide policy change in 2013, offering free maternity services to all women to address this concern. Here, we explore the impact of this policy change on maternal and neonatal outcomes specific to the hypertensive disorders of pregnancy.

\section{Methods}

We conducted a retrospective cross-sectional chart review of women discharged or deceased with a diagnosis of gestational hypertension, preeclampsia, eclampsia or HELLP syndrome at a tertiary referral center in western Kenya one year before (June 1, 2012-May 31, 2013) and one year after (June 1, 2013May 31,2014$)$ free maternity services were introduced at public facilities across the country. Demographic information, obstetric history, medical history, details of the current pregnancy, diagnosis on admission and at discharge, antepartum treatment, maternal outcomes, and neonatal outcomes were collected and compared.

\section{Results}

There were more in hospital deliveries after policy change was introduced. The proportion of women diagnosed with a hypertensive disorder of pregnancy was higher in the year before free maternity care although there was a statistically significant increase in the proportion of women diagnosed with gestational hypertension after policy change. Among those women diagnosed with hypertensive disorders, there was no difference in the proportion who developed obstetric or medical complications. Of concern, there was a statistically significant increase in the proportion of women dying as a result of their condition after policy change. There was a statistically significant increase in the use of magnesium sulfate for seizure prophylaxis. There was no overall difference in the use of anti-hypertensives between groups and no overall difference in the proportion of women who received dexamethasone for fetal lung maturity.

\section{Conclusions}

Free maternity services, however necessary, are insufficient to improve maternal and neonatal outcomes related to the hypertensive disorders of pregnancy at a tertiary referral center in western Kenya. Multiple complementary strategies acting in unison are urgently needed. 


\section{Background}

Preeclampsia/eclampsia continues to be a leading contributor to global maternal and neonatal mortality. ${ }^{1}$ Hemorrhage, sepsis and preeclampsia together account for over $50 \%$ of direct maternal deaths. ${ }^{2}$ Without access to timely, appropriate medical care, up to 63,000 women die and 500,000 pregnancies end in stillbirth as a result of preeclampsia each year. ${ }^{3}$ Ninety-nine percent of these deaths are estimated to occur in resource-limited settings. ${ }^{3}$ Preventing these outcomes requires early diagnosis, prompt referral, and skilled antenatal and delivery care including administration of magnesium sulfate. In Kenya, fewer than $30 \%$ of women attend the recommended four antenatal visits and only approximately $60 \%$ deliver in health care facilities. ${ }^{3,4}$ Current efforts to reduce complications from preeclampsia/eclampsia in LMIC have largely focused on devising clinical risk scores and creating pointof-care tests that can reliably identify women at risk of developing the disease. Despite these interventions, one of the greatest barriers for women during pregnancy and at the time of delivery remains the cost of care. ${ }^{5}$

Preventable pregnancy-related deaths have largely been attributed to delivery without a skilled birth attendant, generally considered a less expensive alternative to hospital-based care. ${ }^{6}$ To address this and accelerate progress towards universal health coverage, Kenya joined other African countries in the abolishment of delivery fees in all public health facilities through a presidential directive effective June 1st 2013. ${ }^{6}$ With little warning, public health facilities became eligible for reimbursement for every woman presenting in labour, or who delivers during admission, through a capitation fund provided by the Ministry of Health. ${ }^{6}$ Few facilities were prepared for the large influx of patients. Initial analysis suggests a $30 \%$ sustained increase in the number of deliveries in public health facilities in the 24 months following policy change. ${ }^{6}$ Similar policy changes in Ghana, Senegal, Tanzania, and Burkina Faso have all been associated with significant increases in institutional delivery rates. ${ }^{7,8}$

With an increase in the number of women able to access care for delivery (and for the treatment of pregnancy related complications, by extension), we hypothesized that a higher proportion would be diagnosed with and treated for hypertensive disorders of pregnancy. We predicted that improved access would encourage women to present earlier in the course of their disease. This would facilitate better care and prevent severe complications. We hypothesized that free access to magnesium sulfate would decrease the proportion of women who develop eclampsia after presentation to hospital.

Conversely, a significant increase in the number of women presenting for care without a proportional increase in hospital resources was hypothesized to potentially overwhelm the system. This could ultimately negatively impact the care all women receive. The overall effect of free maternity services on maternal and neonatal mortality is therefore difficult to estimate. By exploring outcomes related to a specific disease, we will be better able to understand perinatal care at a systems level and identify major gaps in service provision. 
Without population-based tools to track health outcomes for all pregnant women in Kenya, we focused on our institution, Moi Teaching and Referral Hospital (MTRH), to assess the impact of policy change on outcomes specific to the hypertensive disorders of pregnancy. As the second-largest referral hospital in the country and serving a population of over 3.5 million, MTRH provided a unique platform to conduct this study. It is often the final institution in a chain of referrals for women with pregnancy-related complications. In a study conducted at MTRH prior to this policy change, eclampsia accounted for $22 \%$ of all maternal deaths. ${ }^{9}$ An additional $7 \%$ of all neonatal deaths were directly attributable to eclampsia and $38 \%$ to prematurity, which is often associated with severe preeclampsia. ${ }^{9}$

\section{Methods}

\section{Aim}

Our primary objective is to determine the effect of free maternity care on the incidence of the hypertensive disorders of pregnancy diagnosed at a tertiary referral center in Kenya and the impact it has on related maternal and newborn outcomes. We will compare data at our institution in the year before and the year after the introduction of free maternity services at public facilities across Kenya. We will evaluate the adherence to treatment protocols in accordance with World Health Organization recommendations. Our broader objective is to identify areas for improvement in the management of the hypertensive disorders of pregnancy at our institution and thereby decrease maternal and neonatal mortality.

\section{Study Design}

We conducted a retrospective cross-sectional chart review of women discharged or deceased with a diagnosis of gestational hypertension, preeclampsia, eclampsia or HELLP syndrome at MTRH one year before (June 1, 2012-May 31, 2013) and one year after (June 1, 2013-May 31, 2014) implementation of free maternity services at public facilities. We used data collected by the institution to track the total number of antenatal admissions/deliveries during both time periods to compare the incidence of disease between the groups.

\section{Study Population}

The hospital records office maintains an electronic database of discharge diagnoses for all patients. To ensure all eligible women were included, we identified those with gestational hypertension, preeclampsia, eclampsia or HELLP syndrome and cross-referenced this list with women identified as having received antihypertensives and/or magnesium sulfate from the hospital's pharmacy database. Based on the list generated using these criteria, patient charts were reviewed by two trained research assistants.

Gestational hypertension was defined as blood pressure $>/=140 / 90$ without proteinuria, preeclampsia was defined as blood pressure in the range of 140-159/90-109 and any proteinuria on urine dipstick, severe preeclampsia was defined as blood pressure $>/=160 / 110$ and any proteinuria on urine dipstick, HELLP syndrome was defined as biochemical evidence of hemolysis, elevated liver enzymes and low platelets with or without symptoms of preeclampsia, and eclampsia was defined as any of the above 
with otherwise unexplained seizures. These definitions are based on the ACOG guideline on High Blood Pressure in Pregnancy published in 2000 and were the ones used at MTRH at the time of our chart review. ${ }^{10}$ More recent literature would suggest that any proteinuria measured on urine dipstick is predictive of worse maternal outcomes in low resource settings and its measurement remains useful for risk stratification. ${ }^{11}$

\section{Statistical Analysis}

Demographic information, obstetric history, medical history, details of the current pregnancy, diagnosis on admission and at discharge, antepartum treatment, maternal outcomes, and neonatal outcomes were all collected. Infant charts were retrieved when admission to the Newborn Unit (NBU) occurred following delivery. Any patient chart in which death was recorded was reviewed independently by three members of the study team to ascertain the cause of death.

Sample size was calculated using a 50\% difference in the rate of diagnosis of eclampsia among women presenting with a hypertensive disorder in pregnancy pre- and post-policy change. Based on a survey of the hospital's database, we estimated that $10 \%$ of pregnant women at MTRH were discharged with a diagnosis of preeclampsia. This is in keeping with the estimated global prevalence of preeclampsia of 5$7 \%$ and the expected caseload at a tertiary referral hospital. ${ }^{1}$ Using Pocock's formula, we determined that we needed 432 women in each group (pre and post-policy change) to detect a $50 \%$ difference in event rate, with $80 \%$ power and $5 \%$ significance level.

Data was entered into REDCap Version 6.10.2 and analyzed using STATA/IC 12. The data were grouped into two periods spanning the year before the introduction of free maternity services (June 1, 2012-May 31,2013 ) and the year after its introduction (June 1, 2013-May 31, 2014) and presented in tables comparing the groups. Descriptive data including means for continuous variables and proportions for categorical variables were compared using t-test and chi-square tests, respectively with $p<0.05$ considered significant. When the expected frequency was less than 5 , Fisher's exact $p$-value was used instead of the chi-square $p$-value.

\section{Results}

There were a total of 19,374 deliveries at MTRH between June 1, 2012 and May 31, 2014. There were 8,472 deliveries before and 10,902 deliveries after the introduction of free maternity services. The proportion of women diagnosed with a hypertensive disorder of pregnancy was higher in the year before free maternity care was introduced $(12.8 \%$ vs $11.4 \%, p=0.0029)$. Overall, $60 \%$ of the charts belonging to women with a hypertensive disorder of pregnancy were available for data collection. Our analysis included 412 patients before, and 623 after the policy change. Please refer to Figure 1 for further details.

As shown in Table 1, demographic characteristics between groups. The mean patient age was $27.4 \pm$ SD 6 and the median parity was 1 . There was no significant difference in the proportion of adolescents (aged 10-19 as per the WHO) or women > 35 years of age who presented for care. Most women reported being 
married (80\%) with the most commonly reported form of employment being work within the home (38\%). There was no difference in distance traveled to hospital or in the referral rate from smaller, community facilities. There was no difference in the proportion of women with a history of chronic hypertension or diagnosis of preeclampsia or eclampsia in a previous pregnancy before and after the policy change.

\section{Table 1: Background demographic and health characteristics}




\begin{tabular}{|c|c|c|c|}
\hline Demographic & Before $(n=428)$ & After $(n=623)$ & $P$ value \\
\hline Age (mean) & 27.4 (SD 6.06) & 27.4 (SD 6.06) & 0.945 \\
\hline Age $<20$ & $32(7.5 \%)$ & $38(6.1 \%)$ & 0.379 \\
\hline Age $>35$ & $47(11.0 \%)$ & $67(10.8 \%)$ & 0.907 \\
\hline Parity & $216(49.5 \%)$ & $311(49.6 \%)$ & 1.000 \\
\hline 0 & $179(41.1 \%)$ & $257(41.0 \%)$ & \\
\hline $1-4$ & $41(9.4 \%)$ & $59(9.4 \%)$ & \\
\hline \multicolumn{4}{|l|}{$4+$} \\
\hline Distance from Hospital (km) & 23.6 (SD 37.6) & 25.6 (SD 37.1) & 0.415 \\
\hline Referred from Periphery & $154(40.3 \%)$ & $242(42.6 \%)$ & 0.481 \\
\hline GA (at presentation) & $17(4.6 \%)$ & $28(5.3 \%)$ & 0.619 \\
\hline$<28$ weeks & $61(16.4 \%)$ & $107(20.2 \%)$ & 0.151 \\
\hline $28-34$ & $293(79.0 \%)$ & $393(74.5 \%)$ & 0.118 \\
\hline \multicolumn{4}{|l|}{ >34 weeks } \\
\hline Education & $2(0.5 \%)$ & $3(0.5 \%)$ & \multirow[t]{5}{*}{$0.744^{f}$} \\
\hline None & $40(9.2 \%)$ & $53(8.5 \%)$ & \\
\hline Primary & $148(34 \%)$ & $230(36.7 \%)$ & \\
\hline Secondary + Post-Secondary & $246(56.4 \%)$ & $341(54.3 \%)$ & \\
\hline \multicolumn{3}{|l|}{ Missing } & \\
\hline Marital status & $78(17.8 \%)$ & $112(17.9 \%)$ & \multirow[t]{5}{*}{$0.425^{f}$} \\
\hline Single & $351(80.5 \%)$ & $505(80.5 \%)$ & \\
\hline Married & $2(0.5 \%)$ & $0(0.0 \%)$ & \\
\hline Separated/Divorced & $5(1.2 \%)$ & $10(1.6 \%)$ & \\
\hline Missing & & & \\
\hline $\begin{array}{l}\text { Medical History } \\
\text { cHTN }\end{array}$ & $15(0.03 \%)$ & $25(0.04 \%)$ & 0.389 \\
\hline RHD & $1(0.0 \% \%)$ & $0(0.0 \%)$ & 0.230 \\
\hline Diabetes & $1(0.2 \%)$ & $2(0.3 \%)$ & $1.000^{f}$ \\
\hline Renal & $2(0.5 \%)$ & $0(0.0 \%)$ & $0.168^{f}$ \\
\hline
\end{tabular}




\begin{tabular}{|lccc|}
\hline Neurologic & $7(1.6 \%)$ & $6(1.0 \%)$ & 0.344 \\
\hline HIV & $6(1.4 \%)$ & $3(0.5 \%)$ & $0.172^{f}$ \\
History of & $17(3.9 \%)$ & $27(4.3 \%)$ & 0.743 \\
PET & $22(9.4 \%)$ & $25(7.3 \%)$ & 0.367 \\
Eclampsia & $4(0.92 \%)$ & $2(0.32 \%)$ & $0.803^{f}$ \\
\hline
\end{tabular}

All background characteristics included for evaluation were similar between groups.

cHTN = chronic hypertension, $R H D=$ rheumatic heart disease, $P E T=$ preeclampsia

f Fisher's exact

${ }^{m}$ Median test

* Denominator is women with parity $>0$

As illustrated in Table 2, there was no change in the proportion of women delivering live births. In both groups, nearly all women delivered at MTRH (96\%) (as opposed to a peripheral facility, before referral or in transit), 43\% underwent induction of labor, delivered vaginally (64\%) and to a singleton pregnancy (93\%). After the policy change, there was a trend towards increased preterm delivery but this did not reach statistical significance. Of note, there was no change in the median time from admission to delivery ( 1 day).

Table 2: Admission and delivery outcomes among women with live births 


\begin{tabular}{|c|c|c|c|}
\hline & Before $(n=437)$ & After $(n=626)$ & $P$ value \\
\hline Live births & $394(90.2 \%)$ & $551(88.0 \%)$ & 0.274 \\
\hline Stillbirths & $43(9.8 \%)$ & $75(12 \%)$ & 0.391 \\
\hline GA (at delivery) mean & $4(1.2 \%)$ & $6(1.3 \%)$ & 0.928 \\
\hline$<28$ weeks & $43(13.0 \%)$ & $77(16.5 \%)$ & 0.179 \\
\hline 28-34 weeks & $280(84.9 \%)$ & $467(80.9 \%)$ & 0.153 \\
\hline \multicolumn{4}{|l|}{ >34 weeks } \\
\hline \multirow{2}{*}{$\begin{array}{l}\text { Median Interval time from admission to delivery, } \\
\text { range (days) }\end{array}$} & $1(0-32)$ & $1(0-46)$ & 0.240 \\
\hline & $\begin{array}{l}\text { Mean }=2.1 \pm \\
4.6\end{array}$ & $\begin{array}{l}\text { Mean }=2.2 \pm \\
4.6\end{array}$ & 0.754 \\
\hline Location of delivery & $367(96.8 \%)$ & $518(95.9 \%)$ & \multirow[t]{4}{*}{$0.578^{f}$} \\
\hline MTRH & $8(2.1 \%)$ & $18(3.3 \%)$ & \\
\hline Hospital (other then MTRH) & $4(1.1 \%)$ & $5(5.3 \%)$ & \\
\hline \multicolumn{3}{|l|}{ Home/Transit) } & \\
\hline Type of delivery & $166(42.2 \%)$ & $245(44.7 \%)$ & 0.493 \\
\hline Induction & $239(63.1 \%)$ & $347(64.1 \%)$ & \multirow[t]{5}{*}{$0.218^{f}$} \\
\hline SVD & $6(1.6 \%)$ & $8(1.5 \%)$ & \\
\hline Assisted VD & $134(35.3 \%)$ & $182(33.6 \%)$ & \\
\hline CS & $0(0.0 \%)$ & $4(0.7 \%)$ & \\
\hline \multicolumn{3}{|l|}{ Not recorded } & \\
\hline Type of Fetuses delivered & $364(92.9 \%)$ & $520(94.5 \%)$ & \multirow[t]{4}{*}{$0.566^{f}$} \\
\hline Singleton & $27(6.9 \%)$ & $28(5.1 \%)$ & \\
\hline Twins & $0(0.0 \%)$ & $1(1.2 \%)$ & \\
\hline \multicolumn{3}{|l|}{ Triplets } & \\
\hline \multirow[t]{2}{*}{ Birth weight } & $\begin{array}{l}2750.9(700- \\
4350)\end{array}$ & $\begin{array}{l}2685.6(270- \\
4850)\end{array}$ & \multirow{2}{*}{$\begin{array}{l}0.03 \\
0.096\end{array}$} \\
\hline & $\begin{array}{l}\text { Mean } 2652 \pm \\
702.7\end{array}$ & $\begin{array}{l}\text { Mean } 2568 \pm \\
769.6\end{array}$ & \\
\hline $\begin{array}{l}\text { Maternal median and range laboratory values } \\
\text { during admission }\end{array}$ & $\begin{array}{l}13.3(3.0- \\
17.1)\end{array}$ & $11.6(3.2-19.6)$ & $0.369^{m}$ \\
\hline Hemoglobin (mg/dl) & 201.5 (13-640) & $186(6-618)$ & $0.034^{m}$ \\
\hline Platelets & & $\begin{array}{l}15.2(1.9- \\
1,399.5)\end{array}$ & $0.102^{m}$ \\
\hline
\end{tabular}




$\begin{array}{llll}\text { ALT (IU) } & 14.3(0.4- & 27.2(6.0- & 0.403 \mathrm{~m} \\ \text { AST }(\mathrm{IU}) & 2,362) & 3,977.4) & <0.001 \\ \text { Creatinine }(\mathrm{mg} / \mathrm{dL}) & 25.7(10.1- & 66(1.4-1,669) & \mathrm{m} \\ & 3,112.8) & & \\ & 58(4.4-873) & & \\ \text { Maternal median and range BP recorded during } & 148.5(109.5- & 150(119.5- & 0.017^{\mathrm{m}} \\ \text { admission } & 221) & 205) & <0.001^{\mathrm{m}} \\ \text { Systolic }(\mathrm{mmHg}) & 95(39.5-160) & 98(48.5-160) & \\ \text { Diastolic }(\mathrm{mmHg}) & & & \end{array}$

*due to facility record-keeping standards, detailed delivery information about stillbirth cases was not uniformly available and therefore these cases were not included for analysis here (see Table 5).

$S V D=$ spontaneous vaginal delivery, assisted VD = assisted vaginal delivery (with Kiwi vacuum), CS = cesarean section

f Fisher's exact

m Median test

\section{Scope of Hypertensive Disorders}

Table 3 outlines the distribution of hypertensive disorders between groups. Among those women diagnosed with a hypertensive disorder of pregnancy, there was an upward trend in the proportion of women diagnosed with eclampsia and HELLP after policy change, although this did not reach statistical significance. There was a statistically significant increase in the proportion of women diagnosed with gestational hypertension ( $4.1 \%$ vs $8.1 \%, \mathrm{p}=0.009)$ after the policy change.

Table 3: Distribution of Hypertensive Disorders (as previously defined - see Background)

\begin{tabular}{|llll|}
\hline Hypertensive disorders & Before $(\mathbf{n}=360)$ & After $(\mathbf{n}=529)$ & P value \\
\hline gHTN & $18(4.1 \%)$ & $51(8.1 \%)$ & 0.009 \\
\hline PET & $199(45.6 \%)$ & $266(42.4 \%)$ & 0.298 \\
\hline Severe PET & $95(21.8 \%)$ & $114(18.2 \%)$ & 0.146 \\
\hline Eclampsia & $33(7.6 \%)$ & $63(10.1 \%)$ & 0.165 \\
\hline HELLP & $15(3.4 \%)$ & $35(5.6 \%)$ & 0.105 \\
\hline
\end{tabular}

gHTN = gestational hypertension, $P E T=$ preeclampsia 
After policy change, there was a statistically significant decrease in the number of women with a hypertensive disorder of pregnancy who presented to hospital for concerns specific to labor and delivery (labor pains and/or suspected rupture of membranes) (54.4\% vs $45.6 \%, p=0.015$ and $11.7 \%$ vs $6.5 \%$, $p=0.005$, respectively), as illustrated in Table 4 . It should be noted that many women presented with more than one complaint both before and after the policy change.

\section{Table 4: Chief Complaint(s) at Presentation to MTRH}

\begin{tabular}{|c|c|c|c|}
\hline $\begin{array}{l}\text { Chief Complaint(s) at Presentation of Women Diagnosed with } \\
\text { Hypertensive Disorder of Pregnancy }\end{array}$ & $\begin{array}{l}\text { Before } \\
(n=436)\end{array}$ & $\begin{array}{l}\text { After } \\
(n=627)\end{array}$ & $\begin{array}{l}P \\
\text { value }\end{array}$ \\
\hline Lower abdominal pains/Labor & $\begin{array}{l}237 \\
(54.4 \%)\end{array}$ & $\begin{array}{l}286 \\
(45.6 \%)\end{array}$ & 0.015 \\
\hline Drainage of liquor/SROM & $\begin{array}{l}51 \\
(11.7 \%)\end{array}$ & $\begin{array}{l}41 \\
(6.5 \%)\end{array}$ & 0.005 \\
\hline Headache & $\begin{array}{l}131 \\
(30.0 \%)\end{array}$ & $\begin{array}{l}216 \\
(34.5 \%)\end{array}$ & 0.314 \\
\hline Swelling/Edema & $\begin{array}{l}123 \\
(28.3 \%)\end{array}$ & $\begin{array}{l}177 \\
(28.3 \%)\end{array}$ & 0.965 \\
\hline Epigastric/RUQ pain & $\begin{array}{l}71 \\
(16.3 \%)\end{array}$ & $\begin{array}{l}82 \\
(13.1 \%)\end{array}$ & 0.121 \\
\hline Decreased Fetal movement & $\begin{array}{l}44 \\
(10.1 \%)\end{array}$ & $\begin{array}{l}62 \\
(9.9 \%)\end{array}$ & 0.659 \\
\hline Visual changes & $43(9.9 \%)$ & $\begin{array}{l}56 \\
(8.9 \%)\end{array}$ & 0.817 \\
\hline Nausea +/- vomiting & $20(4.6 \%)$ & $\begin{array}{l}34 \\
(5.4 \%)\end{array}$ & 0.799 \\
\hline
\end{tabular}

SROM = spontaneous rupture of membranes

\section{Adverse Outcomes}

Among those women diagnosed with hypertensive disorders, there was no difference in the proportion who developed obstetric or medical complications, including severe postpartum hemorrhage, disseminated intravascular coagulopathy (DIC), renal failure requiring dialysis, pulmonary edema, or stroke between the groups. Of major concern, there was a statistically significant increase in the proportion of women dying as a result of their condition $(1.2 \%$ vs $3.03 \%, p=0.042)$ after policy change.

There was a significantly higher incidence of preterm births between 28-34 weeks when stillbirths were included in the analysis $(13.5 \%$ vs $21.6 \%, p=0.003)$ after policy change. There were also more neonates with Apgar scores less than 7 at 1 minute $(23 \%$ vs $29 \%, p=0.048)$. There was a trend towards higher admission rates to the NBU, stillbirths, and neonatal deaths after policy change, although these 
differences did not reach statistical significance. There was no difference in fetal/neonatal adverse outcomes among women with multiple gestation (data not shown). Please refer to Table 5 for full details of maternal and neonatal outcomes.

Table 5: Significant Maternal and Neonatal Outcomes in Women with Hypertensive Disorders of Pregnancy 


\begin{tabular}{|llll|}
\hline Severe Maternal Outcomes & $\mathbf{N}=\mathbf{4 3 6}$ & $\mathbf{N}=\mathbf{6 2 7}$ & P value \\
\hline Death & $\mathbf{5 ( 1 . 1 5 \% )}$ & $\mathbf{1 9}(3.03 \%)$ & $\mathbf{0 . 0 4 2}$ \\
\hline Postpartum Hemorrhage* & $58(13.3 \%)$ & $76(12.1 \%)$ & 0.568 \\
\hline Placental abruption & $10(2.3 \%)$ & $6(1.0 \%)$ & 0.078 \\
\hline Intensive Care Unit admission & $8(1.8 \%)$ & $8(1.3 \%)$ & 0.462 \\
\hline Disseminated intravascular coagulation & $1(0.23 \%)$ & $7(1.12 \%)$ & $0.151^{f}$ \\
\hline Blood transfusion & $45(10.3 \%)$ & $85(13.6 \%)$ & 0.113 \\
\hline Seizure/convulsion & $33(7.6 \%)$ & $63(10.05 \%)$ & 0.165 \\
\hline Need for dialysis & $4(0.92 \%)$ & $13(2.07 \%)$ & 0.139 \\
\hline VTE & $5(1.15 \%)$ & $2(0.32 \%)$ & $0.130^{f}$ \\
\hline Myocardial Infarction & $2(0.5 \%)$ & $1(0.2 \%)$ & $0.571^{f}$ \\
\hline Pulmonary Edema & $3(0.69 \%)$ & $7(1.1 \%)$ & $0.539^{f}$ \\
\hline Stroke & $3(0.69 \%)$ & $4(0.64 \%)$ & $1.000^{f}$ \\
\hline Platelets <50 & $9(2.3 \%)$ & $25(4.5 \%)$ & 0.073 \\
\hline Transaminitis (ALT or AST >70IU) & $55(14.2 \%)$ & $102(19.6 \%)$ & 0.034 \\
\hline Total with an adverse maternal outcome & $147(33.6 \%)$ & $226(36.1 \%)$ & 0.408 \\
\hline Neonatal Outcomes & $12(5.2 \%)$ & $28(4.8 \%)$ & 0.160 \\
\hline Delivery at < 28 completed weeks & $224(55.2 \%)$ & $353(59.9 \%)$ & 0.135 \\
\hline Delivery at 28-34 completed weeks & $46(13.5 \%)$ & $108(21.6 \%)$ & 0.003 \\
\hline Delivery at 34-37 completed weeks & $55(16.2 \%)$ & $91(18.2 \%)$ & 0.455 \\
\hline Low Birth Weight $(<2.5 k g)$ & $141(38.0 \%)$ & $237(42.8 \%)$ & 0.148 \\
\hline NBU Admission & $107(26.4 \%)$ & $189(32.1 \%)$ & 0.052 \\
\hline IUFD/Stillbirth & $41(10.1 \%)$ & $70(11.9 \%)$ & 0.391 \\
\hline Neonatal Death & $13(2.6 \%)$ & 0.434 \\
\hline Total with adverse fetal/neonatal outcomes & & & \\
\hline
\end{tabular}

${ }^{*}$ Postpartum hemorrhage defined as $>500 \mathrm{~mL}$ after vaginal delivery and $>1000 \mathrm{~mL}$ after cesarean section

$V T E=$ venous thromboembolism, IUFD = intra-uterine fetal demise 
${ }^{f}$ Fisher's exact

\section{Pharmacological Management}

As demonstrated in Table 6, there was a statistically significant increase in the use of magnesium sulfate for seizure prophylaxis $(77.3 \%$ vs $84.5 \%, p=0.003)$, mostly among women with gHTN $(20.2 \%$ vs $24 \%$, $p=0.020$ ), after policy change. Of concern, there was a trend towards less use of magnesium sulfate among women with eclampsia and HELLP syndrome. There was no overall difference in the use of antihypertensives between groups. Less than $50 \%$ of women between $28-34$ weeks received dexamethasone for fetal lung maturity in both groups.

Table 6: Pharmacological management of Hypertensive Disorders of Pregnancy

\begin{tabular}{|llll|}
\hline Medication & Before $(\mathbf{n}=\mathbf{4 3 6})$ & After $(\mathbf{n = 6 2 7})$ & P value \\
\hline Magnesium sulfate & $337(77.3 \%)$ & $530(84.5 \%)$ & 0.003 \\
\hline gHTN & $7(30.4 \%)$ & $24(42.1 \%)$ & 0.02 \\
\hline PET & $147(73.9 \%)$ & $231(86.8 \%)$ & 0.298 \\
\hline Severe PET & $81(85.3 \%)$ & $107(93.9 \%)$ & 0.146 \\
\hline Eclampsia & $33(100 \%)$ & $58(92.1 \%)$ & 0.165 \\
\hline HELLP & $14(93.3 \%)$ & $31(88.6 \%)$ & 0.105 \\
\hline & & & \\
\hline Anti-Hypertensives & $433(99.3 \%)$ & $618(98.6 \%)$ & 0.378 \\
\hline Nifedipine (oral) & $374(85.8 \%)$ & $563(89.8 \%)$ & 0.046 \\
\hline Labetalol (IV ) & $80(18.4 \%)$ & $142(22.6 \%)$ & 0.09 \\
\hline Hydralazine (IV and/or oral) & $27(6.2 \%)$ & $26(4.2 \%)$ & 0.132 \\
\hline Methyldopa (oral) & $24(5.5 \%)$ & $27(4.3 \%)$ & 0.369 \\
\hline Atenolol (oral) & $13(3.0 \%)$ & $40(6.4 \%)$ & 0.012 \\
\hline Dexamethasone & & & \\
\hline < 28 weeks & $4(30.9 \%)$ & $5(29.4 \%)$ & 0.929 \\
\hline 28-34 weeks & $24(44.4 \%)$ & $54(48.2 \%)$ & 0.645 \\
\hline 34-37 weeks & $13(22.4 \%)$ & $24(34.8 \%)$ & 0.008 \\
\hline$>37$ weeks & $11(4.7 \%)$ & $5(1.7 \%)$ & 0.045 \\
\hline
\end{tabular}


Medications unrelated to the direct management of a patient's hypertensive disorder were not included in the analysis. Only those patients receiving the correct dose have been included as having received a medication. Of note, oral labetalol is not used at MTRH.

\section{Discussion}

This study aimed to evaluate the impact of free maternity services on maternal and neonatal outcomes related to the hypertensive disorders of pregnancy at a tertiary referral center in western Kenya. There was a significant increase in the number of women presenting to MTRH for delivery after policy change, in keeping with statistics available from other public facilities across the country. ${ }^{5,6}$ There was no change in the proportion of women diagnosed with eclampsia or HELLP syndrome after policy change but there was a significant increase in the proportion of women diagnosed with gestational hypertension. Critically ill women were referred for tertiary level expertise at the same rate, regardless of the cost of care. The increased case rate of mild gestational hypertension was likely because the diagnosis was an incidental finding when women presented in labor.

Significantly fewer women with hypertensive disorders presented with symptoms related to labor (labor pains and/or suspected rupture of membranes) after policy change. It may be inferred that free maternity services are encouraging women with warning signs to present for antenatal care at a facility earlier in the course of their disease/before labor. There was, however, no improvement in the proportion of women who developed serious complications from their condition. Perhaps the most striking finding in this study was that significantly more women died in hospital of their condition after policy change. This may be indicative of the inability of a tertiary care facility to manage the influx of patients after free maternity services were introduced.

There was a significant increase in the proportion of preterm births between 28-34 weeks when stillbirth was included in our analysis, but no change in length of admission before delivery. This may indicate an increase in very sick women presenting at earlier gestations requiring delivery, increased recognition of fetal distress, or an appropriate increase in iatrogenic preterm delivery secondary to improved management of severe maternal disease. There was a trend towards increased admission rate to the NBU, stillbirth, and neonatal death after the policy change but these did not reach statistical significance.

After policy change, more women with gestational hypertension were treated with magnesium sulfate prophylaxis. There was a trend towards less use of magnesium sulfate in women with severe preeclampsia, HELLP syndrome and eclampsia, although this did not reach statistical significance. There was no difference in the use of anti-hypertensives between the groups. Less than $50 \%$ of women received steroids for fetal lung maturity; this did not improve after policy change. These findings indicate that without adequate supplies or training among health care workers, and with a large increase in patient volumes, appropriate management of pregnant women with hypertensive disorders has suffered and was poor to begin with. 
Ours is one of very few studies to evaluate the impact of a demand side financing strategy on outcomes related to a specific maternal diagnosis in LMIC. Preeclampsia is responsible for 1 in 7 maternal deaths worldwide and 1 in 4 perinatal deaths. ${ }^{12}$ It is a major contributor to maternal and neonatal mortality and is worthy of independent investigation to identify gaps in service provision and areas for improvement. Ours is also the only study evaluating the impact of a free maternity services policy on the use of essential medications like magnesium sulfate, antihypertensives, and steroids for fetal lung maturity in LMIC.

Data collection and analysis suffered from a number of limitations. Generalizability of our findings at the national and international level may not be possible given that we sampled only a tertiary referral center in western Kenya. Our cohort may not capture the dynamics of regional factors affecting other health facility management of preeclampsia. A prospective analysis was not possible before policy change because it was announced with little warning and implemented immediately. Therefore, only retrospective analysis could be performed. With more time and resources, more extensive analysis over several years may have revealed subtle trends our study was not powered to detect. As is the case with many chart reviews in LMIC, there was a significant proportion of missing files and incomplete patient data. Although it is unlikely the missing data was biased and changed the outcomes we observed, we cannot be certain. It was not possible to ascertain when patients made the decision to seek medical attention during the course of their disease. This information may have shed light on outcomes that could not have been mitigated or prevented by adequate management on arrival due to very late presentation.

Over the last three decades, governments of LMIC have implemented a variety of strategies to incentivize pregnant women to seek more timely and appropriate care in order to reduce maternal and infant mortality. Evaluations of the impact of user fee removal, including nationwide government policy, have generally found evidence of increased hospital-based delivery rates. ${ }^{13,14,15}$ This is in keeping with our findings and those of others who have reviewed the free maternity services policy in Kenya. 6 Unfortunately, analysis has shown no overall change in the maternal or neonatal mortality rates in Kenya thus far. ${ }^{6,16}$ This is also in keeping with our findings specific to outcomes related to the hypertensive

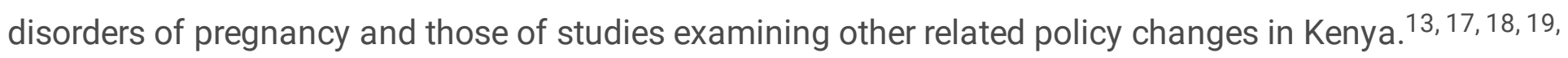
20 In Nigeria, despite adoption of free maternal and child healthcare policies, mortality has remained higher than expected 15 years later. $^{20,21}$

Although a necessary measure, policy change alone does not address many of the other barriers women in LMIC face to access pregnancy-related health care. Free maternity services do not account for the cost of seeking care (transportation) nor the lost opportunity costs related to both seeking and receiving care (lost income generating opportunities). Cost is not the only factor preventing women from utilizing health facilities. In Kenya, preventable maternal and neonatal deaths have also been attributed to the need to travel long distances to reach health centers and cultural practices that encourage women to deliver at home with traditional birth attendants. ${ }^{6}$ 
Poor quality of care at facilities remains a major contributing factor to high maternal and neonatal mortality rates in LMIC. ${ }^{21}$ In Nigeria, identified limitations to care provision have included irregular and interrupted drug supply, health worker absenteeism, poor supervision and monitoring, weak referral systems, absence of written care guidelines, staffing shortages, inconsistent recording and reporting, poorly motivated health workers, mistrust between patients and providers, and inadequate physical infrastructure. ${ }^{21,22,24}$ The majority of these limitations have been described in Kenya following the policy change in 2013 and are highlighted by the lack of improved outcomes observed in our study. ${ }^{6}$

In 2013, a service readiness availability map was conducted in Kenya when the policy change was implemented. Results demonstrated that only $28 \%$ of facilities in the country had essential medicines for pregnancy-related emergencies, including magnesium sulfate. ${ }^{1,25}$ This highlights the inability of public health facilities to adequately manage even the basic principles of preeclampsia care and reflects our findings. Reliable access to life saving medication and equipment is evidently a major barrier impeding improvement to maternal and neonatal outcomes that must be urgently addressed.

Initiatives focusing on risk stratification tools and early identification of women at risk of developing preeclampsia in LMIC often fail to consider the myriad of barriers women face after diagnosis. The data presented here suggest that no one strategy targeting only one barrier faced by such women will be sufficient to affect change. Failure to measure improved maternal or neonatal outcomes does not necessarily indicate that an intervention has failed, but instead illuminates other service gaps to be addressed. At MTRH, a Maternal Fetal Medicine clinical fellowship was initiated in 2019, in collaboration with the University of Toronto, to help address a number of the facility-based factors identified after policy change. The added local health provider training, education and supervision are hoped to have a sustainable positive impact on patient care. Data collection remains ongoing.

\section{Conclusion}

In summary, this study demonstrates that more women presented to a tertiary care facility for labor and delivery in western Kenya after the introduction of free maternity services. There was no increase in the proportion of women who presented with hypertensive disorders of pregnancy and no improvement in related outcomes. There was also no improvement in the use of essential medications. This demonstrates that in isolation, free maternity services, however necessary, are insufficient to improve preeclampsia related outcomes in Kenya. Multiple complementary strategies acting in unison are urgently needed.

\section{Abbreviations}

LMIC - Low and Middle Income Countries

MTRH - Moi Teaching and Referral Hospital 
HELLP - Hemoloysis, Elevated Liver Enzymes, Low Platelets

ACOG - American College of Obstetricians and Gynecologists

NBU - Newborn Unit

cHTN - Chronic Hypertension

RHD - Rheumatic Heart Disease

PET - Preeclampsia

SVD - Spontaneous Vaginal Delivery

Assisted VD - Assisted Vaginal Delivery

CS - Cesarean Section

SROM - Spontaneous Rupture of Membranes

DIC - Disseminated Intravascular Cooagulopathy

VTE - Venous Thromboembolism

IUFD - Intra-uterine Fetal Demise

gHTN - Gestational Hypertension

\section{Declarations}

\section{Ethics approval and consent to participate}

All data was collected in accordance with the Declaration of Helsinki. This study was approved by the Institutional Review Ethics Committee (IREC) at MTRH/Moi University (IREC/2014/259), the Review Ethics Board (REB) at the University of Toronto (\#31133), and the Institutional Review Board at Indiana University before data collection commenced. A waiver of individual informed consent was granted by the aforementioned Ethics Committees because the research involved minimal risk to the subjects, the waiver was not felt to adversely affect the rights and welfare of the subjects, and the research could not practically be carried out without the waiver (mainly due to large sample size and anticipated significant loss to follow up). Results are reported according to STROBE guidelines for observational research.

\section{Consent for publication}

Not applicable 
- The raw dataset used during the current study is available from the corresponding author on reasonable request.

- All data analysed are included in this published article

\section{Competing interests}

The authors declare that they have no competing interests.

\section{Funding}

Not applicable.

\section{Authors' contributions}

Conception/design of the work: ACD, JT, CB

Data collection: JT, JM, VK

Data analysis and interpretation: $\mathrm{MB}, \mathrm{COB}, \mathrm{ACD}, \mathrm{WK}, \mathrm{JT}, \mathrm{BP}$

Drafting the article: $\mathrm{MB}$

Critical revision of the article: $M B, C B, W K, H M, J M, J T, A C D$

Final approval of the version to be published: $\mathrm{CB}, \mathrm{ACD}$

All authors read and approved the final manuscript.

Correspondence to: marie.buitendyk@medportal.ca

\section{Acknowledgements}

Not applicable.

\section{References}

1. WHO recommendations for prevention and treatment of preeclampsia and eclampsia. Geneva: World Health Organization; 2011.

2. Say, L., et al. (2014). "Global causes of maternal death: a WHO systematic analysis." The Lancet Global Health 2(6): e323-e333.

3. WHO recommendations: Policy of interventionist versus expectant management of severe preeclampsia before term. Geneva: World Health Organization; 2018. Licence: CC BY-NC-SA 3.0 IGO.

4. Kenya Demographics and Health Survey. Nairobi: Kenya; 2015. 
5. Lang'at, E., et al. (2019). "Effects of implementing free maternity service policy in Kenya: an interrupted time series analysis." BMC Health Serv Res 19(1): 645.

6. Gitobu, C. M., et al. (2018). "Satisfaction with Delivery Services Offered under the Free Maternal Healthcare Policy in Kenyan Public Health Facilities." J Environ Public Health 2018: 4902864.

7. Kruk, M. E. and L. P. Freedman (2008). "Assessing health system performance in developing countries: a review of the literature." Health Policy 85(3): 263-276.

8. Brazier, E., et al. (2009). "Improving poor women's access to maternity care: Findings from a primary care intervention in Burkina Faso." Soc Sci Med 69(5): 682-690.

9. Yego, F., et al. (2013). "A retrospective analysis of maternal and neonatal mortality at a teaching and referral hospital in Kenya." Reprod Health 10: 13.

10. "Diagnosis and management of preeclampsia and eclampsia."ACOG Practice Bulletin. (2002). 99(1): 159-168.

11. Magee, L. A., et al. (2014). "Diagnosis, evaluation, and management of the hypertensive disorders of pregnancy." Pregnancy Hypertens 4(2): 105-145.

12. Hodgins, A. (2015). "Pre-eclampsia as underlying cause for perinatal deaths: Time for action." GHSP (3) 4: 525-527.

13. Morgan, L., et al. (2013). "Financial incentives and maternal health: Where do we go from here?" J Health Popul Nutr 31(4 Suppl 2): 58-522.

14. Edoka, I., et al. (2016). "Free health care for under-fives, expectant and recent mothers? Evaluating the impact of Sierra Leone's free health care initiative." Health Econ Rev 6(1): 19.

15. Bergevin, Y., et al. (2015). "Towards ending preventable maternal deaths by 2035." Semin Reprod Med 33(1): 23-29.

16. Calhoun, L. M., et al. (2018). "The Effect of the Removal of User Fees for Delivery at Public Health Facilities on Institutional Delivery in Urban Kenya." Matern Child Health J 22(3): 409-418.

17. Burgert, C. R., et al. (2011). "Impact of implementation of free high-quality health care on health facility attendance by sick children in rural western Kenya." Trop Med Int Health 16(6): 711-720.

18. Chuma, J., et al. (2009). "Reducing user fees for primary health care in Kenya: Policy on paper or policy in practice?" Int J Equity Health 8: 15.

19. Obare, F., et al. (2018). "Assessing the community-level impact of a decade of user fee policy shifts on health facility deliveries in Kenya, 2003-2014." Int J Equity Health 17(1): 65.

20. Owiti, A., et al. (2018). "Utilization of Kenya's free maternal health services among women living in Kibera slums: a cross-sectional study." Pan Afr Med J 30: 86.

21. Okonofua, F., et al. (2011). "Advocacy for free maternal and child health care in Nigeria-Results and outcomes." Health Policy 99(2): 131-138.

22. Kana, M. A., et al. (2015). "Maternal and child health interventions in Nigeria: a systematic review of published studies from 1990 to 2014." BMC Public Health 15: 334. 
23. Macha, J., et al. (2012). "Factors influencing the burden of health care financing and the distribution of health care benefits in Ghana, Tanzania and South Africa." Health Policy Plan 27 Suppl 1: i46-54.

24. Ogbuabor, D. C. and O. E. Onwujekwe (2018). "Implementation of free maternal and child healthcare policies: assessment of influence of context and institutional capacity of health facilities in Southeast Nigeria." Glob Health Action 11(1): 1535031.

25. Policy Proposal on the Presidents' Initiative on Free Maternal Health Services in Kenya. Nairobi: Ministry of Health; 2013.

\section{Figures}




\section{Total Deliveries at MTRH}

19374

Before free maternity care

8472

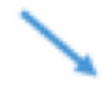

After free maternity care

Total Cases Hypertensive Disorders of

Pregnancy

2333

Before free maternity care

1086

After free maternity care

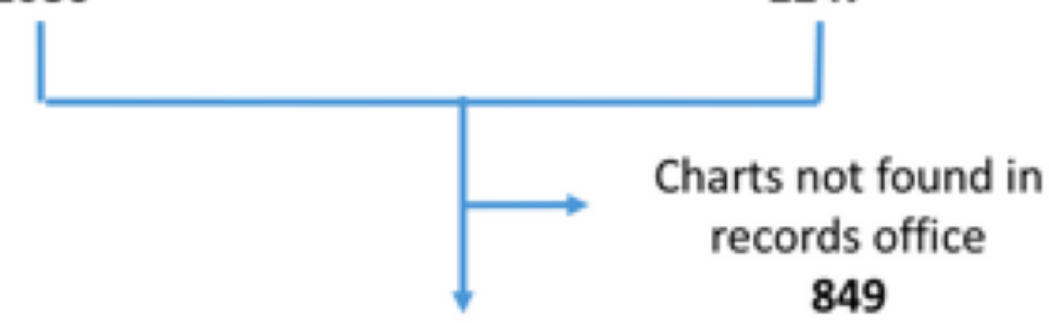

Charts retrieved from records office

\section{4}

Charts entered in the study

Charts excluded after

review

414

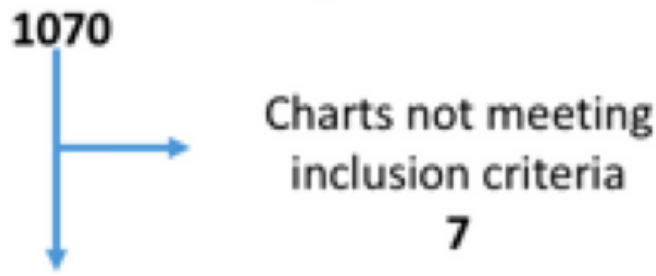

Charts included for analysis

1063

Before free maternity care

436
After free maternity care

627

\section{Figure 1}

Patient selection map - Total number of deliveries at MTRH during the 2 year study period was 19,374. Of these, 2,333 were identified as having a hypertensive disorder of pregnancy from the electronic discharge database and pharmacy records at the hospital. 1,484 of these eligible patient charts were located in the records office and, after review, 1063 were included in our analysis. 